\title{
Where's WALY? : A proof of concept study of the 'wellbeing adjusted life year' using secondary analysis of cross-sectional survey data
}

Rebecca Johnson, David Jenkinson, Chris Stinton, Sian Taylor-Phillips, Jason Madan, Sarah Stewart-Brown and Aileen Clarke*

\begin{abstract}
Background: The Quality-Adjusted Life Year (QALY) is a measure that combines life extension and health improvement in a single score, reflecting preferences around different types of health gain. It can therefore be used to inform decision-making around allocation of health care resources to mutually exclusive options that would produce qualitatively different health benefits. A number of quality-of-life instruments can be used to calculate QALYs. The EQ-5D is one of the most commonly used, and is the preferred option for submissions to NICE (https://www.nice.org.uk/process/pmg9/). However, it has limitations that might make it unsuitable for use in areas such as public and mental health where interventions may aim to improve well-being. One alternative to the QALY is a Wellbeing-Adjusted Life Year. In this study we explore the need for a Wellbeing-Adjusted Life Year measure by examining the extent to which a measure of wellbeing (the Warwick-Edinburgh Mental Well-being Scale) maps onto the EQ-5D-3L.
\end{abstract}

Methods: Secondary analyses were conducted on data from the Coventry Household Survey in which 7469 participants completed the EQ-5D-3L, Warwick-Edinburgh Mental Well-being Scale, and a measure of self-rated health. Data were analysed using descriptive statistics, Pearson's and Spearman's correlations, linear regression, and receiver operating characteristic curves.

Results: Approximately $75 \%$ of participants scored the maximum on the EQ-5D-3L. Those with maximum EQ-5D-3L scores reported a wide range of levels of mental wellbeing. Both the Warwick-Edinburgh Mental Well-being Scale and the EQ-5D-3L were able to detect differences between those with higher and lower levels of self-reported health. Linear regression indicated that scores on the Warwick-Edinburgh Mental Well-being Scale and the EQ-5D-3L were weakly, positively correlated (with $R^{2}$ being 0.104 for the index and 0.141 for the visual analogue scale).

Conclusion: The Warwick-Edinburgh Mental Well-being Scale maps onto the EQ-5D-3L to only a limited extent. Levels of mental wellbeing varied greatly amongst participants who had the maximum score on the EQ-5D-3L. To evaluate the relative effectiveness of interventions that impact on mental wellbeing, a new measure - a Wellbeing Adjusted Life Year - is needed.

Keywords: EQ-5D, WALY, Wellbeing, WEMWBS

Abbreviations: CHS, Coventry household survey; MSOA, Middle super output areas; QALY, Quality adjusted life year; WALY, Wellbeing adjusted life year; WEMWBS, Warwick-Edinburgh mental well-being scale

* Correspondence: Aileen.Clarke@warwick.ac.uk

Division of Health SciencesWarwick Medical School, University of Warwick

Coventry, Coventry CV4 7AL, UK 


\section{Background}

When making decisions about interventions it is important to consider their effect on both length of life and the quality of that life. One way in which this is achieved is through the use of Quality-Adjusted Life Years (QALY). The most common method of calculating QALYs uses a measure called EQ-5D-3L $[1,2]$ which has been successfully employed to assess the relative effectiveness of a wide range of treatments and interventions. However, there is evidence of ceiling effects in the EQ-5D-3L, with up to $85 \%$ of respondents who have physical health problems reporting maximum scores [3, 4]. Further, there are questions about whether the EQ-5D-3L is appropriate for assessing the impacts of conditions such as hearing loss, age-related macular degeneration, diabetic retinopathy and psychotic disorders. This is because of problems such as failure to detect differences in quality of life between people with different stages of disease severity, and a limited ability to detect improvements in quality of life following interventions [4-9].

Wellbeing is now recognised as a determinant of longevity and an important player in the adoption and maintenance of healthy lifestyles and successful management of chronic illness [10]. The case for improving wellbeing has been made on both health and economic grounds [11]. Interventions to promote mental wellbeing (e.g. parks and gardens, crime reduction, art festivals, cookery clubs, wellbeing festivals, Tai Chi, yoga, sports) may be offered in many different sectors, both public and private and it is important to be able to assess their relative effectiveness compared to interventions offered in the health sector. While researchers have mapped utility of the EQ-5D-3L onto utilities derived from a range of health outcome measures (e.g. SF-6D) [12-14], there has been little research on how to address the cost-utility of interventions aimed at improving mental wellbeing. If wellbeing is a concept that substantially extends existing concepts of health, then a health-related measure of quality-of-life will underestimate the benefit of interventions that improve wellbeing. In a time of austerity, this is clearly an issue for public health commissioning. One approach to address the cost-utility of these types of interventions could be to develop a wellbeing adjusted life year (WALY).

A well-established tool to measure mental wellbeing is the Warwick-Edinburgh Mental Well-being Scale (WEMWBS) [15, 16]. Full details of the WEMWBS are available (www2.warwick.ac.uk/fac/med/research/platform/wemwbs/). In brief, WEMWBS was developed to meet the need for a robust, population-based measure of mental wellbeing to evaluate programmes and monitor mental wellbeing at the population level [15]. WEMWBS has been in use since 2007, it is valid and reliable at the population level [15] and is sensitive to change [17]. Originally validated in English and Scottish populations of people aged 16 and older [15], the scale has now been translated into many different languages and validated in many different cultures [18]. It has been successfully used to measure wellbeing outcomes in a range of health interventions [19-25]. Evidence suggests that users of mental health services and their carers prefer the WEMWBS to other health outcome measures [26].

WEMWBS is gaining momentum as a useful tool in public health practice, particularly since its inclusion as a measure in the Scottish Governments Outcomes Framework [27] and the English Public Health Outcomes Framework [28]. However, there is no underlying research on how to assess the cost-effectiveness of interventions using this tool. In this study we explore the extent to which the WEMWBS and the EQ-5D-3L estimate the health state value of individuals with different levels of mental wellbeing (i.e. whether WEMWBS "maps" onto the EQ-5D-3L) [29]. If mapping is poor, this suggests that there is a need to develop a Wellbeing Adjusted Life Year (WALY).

\section{Methods}

\section{Setting}

Data used in this study come from a survey of residents of Coventry, UK. The health of people who live in Coventry is worse than that for England overall [30]. The gap in life expectancy between men and women is the widest in the West Midlands (approximately 9 years difference between the least deprived and most deprived areas of Coventry). Compared to the average for England, there are more early deaths from cancer, more hospital stays for self-harm, a significantly greater proportion of obese adults and obese children, and significantly lower rates of physical activity among adults in Coventry. Smoking during pregnancy, and alcoholrelated hospital admissions are both higher than the average in England [30]. The Coventry Household Survey (CHS) has measured environment, lifestyle behaviours, and mental wellbeing and health related quality of life (using the EQ-5D-3L) since 2011 [31-33].

\section{Design and participants}

Secondary analysis of cross-sectional survey data taken from the CHS at three time points (2011, 2012, and 2013) [31-33] was conducted. Participants were residents of Coventry who were aged 16 years or older at the time of the survey. There were 3144 participants in the 2011 survey, 2117 participants in the 2012 survey, and 2208 participants in the 2013 survey. No person was surveyed in more than 1 year so data were combined for analyses $(n=7469)$. 


\section{Data collection}

Data were obtained from the CHS which comprises 45 questions in six topic sections: community and neighbourhood, environment and housing, crime and safety, work and training, transport and accessibility, and health and wellbeing. Households were sampled using a stratified sampling approach [34]. The Royal Mail Postcode Address File was used to obtain a full list of addresses in Coventry, which was linked to the Middle Super Output Areas (MSOA). Three postcodes (one random and two numerically next-nearest) were sampled within each of the 42 MSOA to ensure representativeness based on deprivation levels, consistent with the overall population of Coventry. This resulted in 126 primary sampling points. Interviewers used age sampling, asking to speak to the 'household member whose birthday is next'. Approximately 200 additional surveys were conducted around Coventry city centre in order to represent mobile populations. Survey questions were asked by face-to-face interview, with responses recorded by the interviewer, except for WEMWBS which was self-completed. The survey took approximately $20 \mathrm{~min}$ to complete. Data collection was undertaken by the research consultancy firms BMG and MEL using teams of trained, multilanguage interviewers. A $10 \%$ sample of each interviewer's survey batch was checked. A further $10 \%$ of survey participants were contacted to ensure that interviews had taken place as recorded. Data were then anonymised. Data entry, primary coding and cleaning/ consistency checks were undertaken.

\section{Measures}

\section{$E Q-5 D-3 L$}

The EQ-5D-3L is a generic preference-based measure used to assess health-related quality of life and cost effectiveness of health interventions [1]. It measures five dimensions: mobility, self-care, usual activities, pain/discomfort, and anxiety/depression. Each dimension has three levels which are scored as a ' 1 ' (e.g. 'I have no problems in walking about'), '2' (e.g. 'I have some problems in walking about'), or '3' (e.g. 'I am confined to bed'). Each dimension is coded and together comprises an end health state such as '11111' (in this example, no problems in any of the health dimensions are indicated). There are 243 possible health states that are relevant for both clinical and general populations. The EQ-5D-3L utility index uses a time trade-off method For the UK, this is from a sample of 3395 respondents from the general population $[12,13]$ Intra-class coefficients (ICC) of 0.78 have been reported for the Visual Analogue Scale (VAS) and 0.73 for Time Trade Off (TTO) methods, with little non-response effect [12, 35]. The EQ-5D-3L has been used extensively and translated into at least 171 languages (http://www.euroqol.org/). It has been found to be a practical way of measuring and detecting differences in the health states of individuals within general [36, 37] and patient populations [38, 39].

\section{WEMWBS}

WEMWBS is a self-reported measure of mental wellbeing [15]. It is a positively worded 14-item scale covering hedonic (eg I've been feeling cheerful') and eudaimonic (eg 'I have been feeling useful') components of mental wellbeing. For each item, participants can select a response option from 'none of the time' (item score $=1$ ) to 'all of the time' (item score $=5$ ) with a 2 week timeframe. The scale is scored by adding up each item for a total score ranging from 14 to 70 . It has been found valid and reliable (www2.warwick.ac.uk/fac/med/ research/platform/wemwbs/) [15]. WEMWBS was validated using eight scales that incorporated similar concepts or were likely to be associated with mental wellbeing [15]. Data on mental ill health were collected, and social desirability bias was assessed using the Balanced Inventory of Desirable Responding (BIDR) [40]. Content validity was assessed and all response categories were used at least once by respondents, with little evidence of skew within the distributions of each item response. Construct validity was assessed using confirmatory factor analysis with least squares estimation. Both the goodness of fit index (0.91) and adjusted goodness of fit index (0.87) were satisfactory, and the Root Mean Square Error of Approximation was within the desired upper limit (0.0502). Good internal consistency was demonstrated (Cronbach's alpha 0.89 and 0.91 in each sample). There was no evidence of floor or ceiling effects. There were low to moderate correlations with overall health, as measured by EQ-5D VAS $(r=0.43, p<0.01)$ and high correlations with scales measuring aspects of wellbeing or positive affect, such as the Positive and Negative Affect Schedule-Positive Affect Scale, [41] among others (Positive Affect $r=0.71, p<0.01$ ). Equally, there was a negative correlation between Positive and Negative Affect Schedule-Negative Affect Scale and WEMWBS $(r=-0.54, p<0.01)[15]$.

\section{Self-rated health}

Self-rated health (SRH) is a generic health measure used in a range of populations and countries [42-45], and has been has been associated with all-cause mortality [44, 46]. Self-rated health was measured by asking participants "How would you say your health is, in general?". Response options ranged from 'very good' to 'very bad'. $\mathrm{SRH}$ has demonstrated moderate test-retest reliability [43], and has consistent and strong predictive validity with respect to mortality $[47,48]$. 


\section{Statistical analysis}

Analyses were conducted using R, version 3.0.3 [49], with the pROC package [50]. To address health inequalities, analysis was stratified by age, gender, and socioeconomic status. Descriptive statistics and plots were used to explore the distributions of, and the relationships between, WEMWBS and the EQ-5D-3L on each dimension, the visual analogue scale (VAS), and the preference-based index. We assessed the correlation between the EQ-5D-3L VAS and WEMWBS. Floor and ceiling effects were estimated by calculating the proportion of responses at the lowest and highest possible level for each dimension of both the EQ-5D-3L and WEMWBS.

We calculated the area under curve (AUC) of the receiver operating characteristic (ROC) curve to examine whether WEMWBS and the EQ-5D-3L were able to distinguish between participants with 'very good' vs lower self-rated health.

WEMWBS was mapped onto the EQ-5D-3L using methodology derived from Longworth and Rowen [29]. Since we were only interested in whether WEMWBS maps onto the EQ-5D-3L no other variables were included in the model. The model fit for linear models was assessed using the $\mathrm{R}^{2}$ statistic.

\section{Results}

\section{Sample characteristics}

The demographics of the participants, in terms of age, sex and index of multiple deprivation (IMD) quintiles are shown in Table 1.

Table 1 Demographics of participants

\begin{tabular}{lllllll}
\hline & & 2011 & 2012 & 2013 & Total & Percent \\
\hline Age (years) & $16-24$ & 521 & 358 & 342 & 1221 & $16.3 \%$ \\
& $25-34$ & 533 & 378 & 428 & 1339 & $17.9 \%$ \\
& $35-44$ & 462 & 378 & 320 & 1160 & $15.5 \%$ \\
& $45-54$ & 394 & 293 & 310 & 997 & $13.3 \%$ \\
& 55-64 & 346 & 303 & 322 & 971 & $13.0 \%$ \\
& 65-74 & 178 & 266 & 289 & 733 & $9.8 \%$ \\
& 75 and over & 195 & 138 & 178 & 511 & $6.8 \%$ \\
& Not available & 515 & 3 & 19 & 537 & $7.2 \%$ \\
& Male & 1547 & 1020 & 1061 & 3628 & $48.6 \%$ \\
& Female & 1597 & 1095 & 1147 & 3839 & $51.4 \%$ \\
& Not available & 0 & 2 & 0 & 2 & $0.0 \%$ \\
& 1st Quintile & 1179 & 690 & 420 & 2289 & $30.6 \%$ \\
& 2nd Quintile & 798 & 607 & 417 & 1822 & $24.4 \%$ \\
& 5th Quintile & 216 & 148 & 445 & 809 & $10.8 \%$ \\
\hline & 4th Quintile & 559 & 335 & 421 & 1315 & $17.6 \%$ \\
& 4th Quintile & 392 & 337 & 505 & 1234 & $16.5 \%$ \\
& & & & & &
\end{tabular}

\section{Descriptive statistics}

The joint distribution of the EQ-5D-3L and WEMWBS scores is shown in Table 2 and Fig. 1. Marginal distributions show that $74.3 \%$ of the participants had an EQ-5D-3L score of 1, i.e. three out of four participants' EQ-5D-3L scores were clustered at the top most level of the scale where no further measurement would be recorded. This demonstrates a large ceiling effect for the EQ-5D-3L. The marginal distribution of the WEMWBS scores was closer to symmetric, showing a more normal distribution and a wider range of possible WEMWBS scores. The mean EQ-5D-3L score was 0.90, with a standard deviation of 0.23 . The mean WEMWBS score was 52.36, with a standard deviation of 8.85. Correlations between scores on the two measures were $r=0.322$ (95 \% CI: 0.301, 0.342) and $r_{\mathrm{s}}=0.299$ (95 \% CI: 0.275, 0.320). Correlations between WEMWBS and EQ-5D-3L, stratified by age, gender, socioeconomic status, are shown in Table 3.

The joint distribution of WEMWBS with the EQ-5D-3L VAS score is shown in Table 2. The mean EQ-5D-3L VAS score was 77.5, with a standard deviation of 18.4. The median score was 80, with quartiles of 70 and 90. Correlations between the EQ-5D-3L VAS score and WEMWBS were $r=0.375$ (95\% CI: $0.355,0.396)$ and $r_{\mathrm{s}}=0.355(95 \%$ CI: $0.333,0.376)$. The distributions of the WEMWBS scores for each level of each domain of EQ-5D-3L are illustrated in Fig. 2, showing there is a wide spread of WEMWBS scores within each EQ-5D-3L domain.

\section{Construct validity of EQ-5D-3L and WEMWBS Self-rated health}

Nearly one third (30.5\%) of participants reported 'very good' self-rated health. The WEMWBS and EQ-5D-3L were both able to distinguish between participants with 'very good' and less than very good self-rated health: WEMWBS (AUC 0.657 [0.643, 0.670]) and EQ-5D-3L (AUC $0.636[0.628,0.644]$ ). If a participant with very good and a participant with less than very good self-rated health were randomly chosen from the population, WEMWBS has a slightly higher probability than EQ-5D$3 \mathrm{~L}$ of ranking a participant with very good self-rated health higher than one with less than very good self-rated health due to the ceiling effects of EQ-5D-3L as shown by the higher ROC curve to the left of the plot (Fig. 3).

\section{Mapping WEMWBS onto the EQ-5D-3L}

WEMWBS predicted EQ-5D-3L and EQ-5D-3L VAS scores to a limited extent, with adjusted $\mathrm{R}^{2}$ statistics of 0.104 and 0.141 , respectively (Table 4 ).

Linear models indicated that WEMWBS scores explained $10.4 \%$ of the variability in the EQ-5D-3L scores and $14.1 \%$ of the variability in the EQ-5D-3L VAS scores. 
Table 2 Joint distribution of WEMWBS and EQ-5D-3L, and WEMWBS and EQ-5D-3L Visual Analogue Scale

\begin{tabular}{|c|c|c|c|c|c|c|c|c|c|c|c|}
\hline \multirow[b]{2}{*}{ EQ-5D-3L } & \multicolumn{9}{|c|}{ WEMWBS } & \multirow[b]{2}{*}{ Total } & \multirow[b]{2}{*}{$\%$} \\
\hline & $(14,21]$ & $(21,28]$ & $(28,35]$ & $(35,42]$ & $(42,49]$ & $(49,56]$ & $(56,63]$ & $(63,70]$ & NA & & \\
\hline$(-0.6,0]$ & 1 & 13 & 17 & 29 & 29 & 16 & 9 & 0 & 4 & 118 & $1.6 \%$ \\
\hline$(0,0.2]$ & 0 & 9 & 21 & 48 & 43 & 38 & 17 & 4 & 3 & 183 & $2.5 \%$ \\
\hline$(0.2,0.4]$ & 1 & 5 & 6 & 15 & 14 & 9 & 5 & 4 & 1 & 60 & $0.8 \%$ \\
\hline$(0.4,0.6]$ & 3 & 5 & 11 & 54 & 60 & 41 & 11 & 9 & 4 & 198 & $2.7 \%$ \\
\hline$(0.6,0.8]$ & 2 & 11 & 36 & 117 & 268 & 303 & 136 & 39 & 11 & 923 & $12.4 \%$ \\
\hline$(0.8,1)$ & 0 & 7 & 19 & 71 & 115 & 108 & 49 & 23 & 7 & 399 & $5.3 \%$ \\
\hline 1 & 5 & 13 & 41 & 385 & 1032 & 2119 & 1193 & 677 & 87 & 5552 & $74.3 \%$ \\
\hline NA & 1 & 1 & 1 & 7 & 4 & 9 & 3 & 1 & 9 & 36 & $0.5 \%$ \\
\hline Total & 13 & 64 & 152 & 726 & 1565 & 2643 & 1423 & 757 & 126 & 7469 & \\
\hline$\%$ & $0.2 \%$ & $0.9 \%$ & $2.0 \%$ & $9.7 \%$ & $21.0 \%$ & $35.4 \%$ & $19.1 \%$ & $10.1 \%$ & $1.7 \%$ & & \\
\hline \multicolumn{12}{|l|}{ VAS } \\
\hline$(0,50]$ & 5 & 38 & 76 & 189 & 236 & 187 & 66 & 25 & 19 & 841 & $11.3 \%$ \\
\hline$(50,60]$ & 0 & 7 & 14 & 68 & 143 & 120 & 50 & 18 & 4 & 424 & $5.7 \%$ \\
\hline$(60,70]$ & 3 & 2 & 12 & 118 & 217 & 279 & 125 & 48 & 9 & 813 & $10.9 \%$ \\
\hline$(70,75]$ & 0 & 3 & 5 & 41 & 133 & 156 & 80 & 30 & 4 & 452 & $6.1 \%$ \\
\hline$(75,80]$ & 2 & 7 & 20 & 102 & 273 & 524 & 268 & 117 & 23 & 1336 & $17.9 \%$ \\
\hline$(80,85]$ & 0 & 2 & 2 & 33 & 84 & 170 & 105 & 49 & 6 & 451 & $6.0 \%$ \\
\hline$(85,90]$ & 1 & 1 & 5 & 54 & 223 & 523 & 331 & 165 & 18 & 1321 & $17.7 \%$ \\
\hline$(90,95]$ & 0 & 0 & 3 & 17 & 65 & 202 & 115 & 71 & 7 & 480 & $6.4 \%$ \\
\hline$(95,100]$ & 2 & 1 & 6 & 30 & 87 & 252 & 195 & 150 & 9 & 732 & $9.8 \%$ \\
\hline NA & 0 & 3 & 9 & 74 & 104 & 230 & 88 & 84 & 27 & 619 & $8.3 \%$ \\
\hline
\end{tabular}

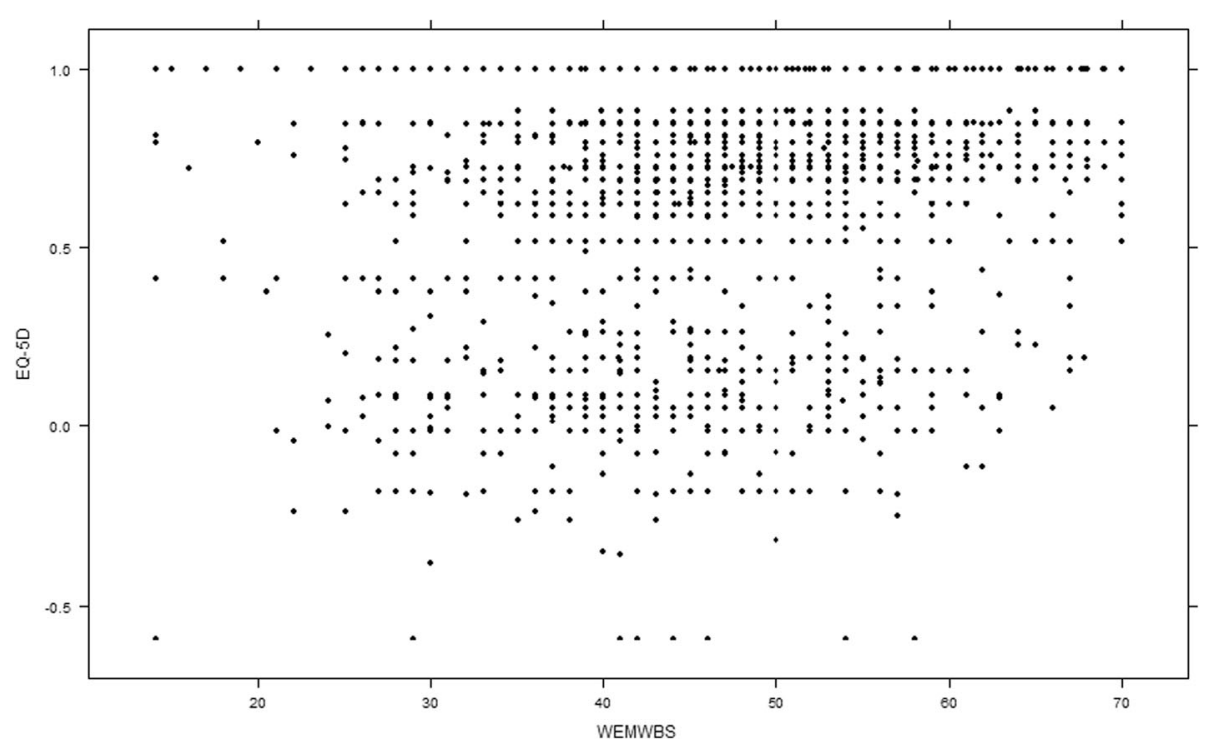

Fig. 1 Scatter plot of WEMWBS and EQ-5D-3L scores 
Table 3 Correlation's between EQ-5D-3L and WEMWBS within each level of the variables age, gender and IMD

\begin{tabular}{|c|c|c|c|c|c|}
\hline & & \multicolumn{2}{|l|}{ Pearson } & \multicolumn{2}{|l|}{ Spearman } \\
\hline & & & $95 \%$ Confidence & & $95 \%$ Confidence \\
\hline & & Estimate & Interval & Estimate & Interval \\
\hline \multirow[t]{7}{*}{ Age } & $16-24$ & 0.238 & $(0.184,0.290)$ & 0.224 & $(0.169,0.278)$ \\
\hline & $25-34$ & 0.300 & $(0.251,0.349)$ & 0.252 & $(0.200,0.303)$ \\
\hline & $35-44$ & 0.312 & $(0.259,0.364)$ & 0.318 & $(0.264,0.371)$ \\
\hline & $45-54$ & 0.344 & $(0.288,0.398)$ & 0.269 & $(0.209,0.327)$ \\
\hline & $55-64$ & 0.389 & $(0.333,0.441)$ & 0.370 & $(0.312,0.425)$ \\
\hline & $65-74$ & 0.313 & $(0.246,0.377)$ & 0.274 & $(0.204,0.341)$ \\
\hline & 75 and over & 0.289 & $(0.206,0.367)$ & 0.350 & $(0.268,0.427)$ \\
\hline \multirow[t]{2}{*}{ Gender } & Male & 0.302 & $(0.272,0.332)$ & 0.275 & $(0.244,0.306)$ \\
\hline & Female & 0.334 & $(0.305,0.362)$ & 0.316 & $(0.286,0.345)$ \\
\hline \multirow[t]{5}{*}{ IMD } & 1st Quintile & 0.308 & $(0.270,0.345)$ & 0.277 & $(0.238,0.316)$ \\
\hline & 2nd Quintile & 0.319 & $(0.277,0.360)$ & 0.296 & $(0.253,0.339)$ \\
\hline & 3rd Quintile & 0.324 & $(0.274,0.372)$ & 0.292 & $(0.240,0.342)$ \\
\hline & 4th Quintile & 0.344 & $(0.293,0.392)$ & 0.298 & $(0.245,0.349)$ \\
\hline & 5th Quintile & 0.336 & $(0.272,0.396)$ & 0.362 & $(0.298,0.422)$ \\
\hline
\end{tabular}

\section{Discussion}

The aim of this study was to assess the extent to which WEMWBS maps onto the EQ-5D-3L. Consistent with previous studies [14], we found a pronounced ceiling effect in the EQ-5D-3L, with nearly three quarters of participants having the maximum score of 1 (i.e. the best possible health-related quality of life). No ceiling effect was observed for WEMWBS. WEMWBS scores spanned the whole range of possible values (14-70), with a mean of 53.9 for participants who had a score of 1 on the EQ5D-3L. This suggests that WEMWBS and the EQ-5D-3L are not measuring the same construct and that there is scope for improving mental wellbeing of individuals who have maximum scores on this quality of life measure. WEMWBS was positively correlated with both the EQ-
5D-3L and the EQ-5D-3L VAS for the sample as a whole and when stratified by age, sex, and socioeconomic status, though this correlation was quite low. Both WEMWBS and the EQ-5D-3L detected differences between those with very good versus other levels of selfreported health, but neither measure was especially good at detecting these differences. This is not surprising as the constructs that they are measuring are not identical. WEMWBS explained a very limited amount of the variability of the EQ-5D-3L and the EQ-5D-3L could not assess with any precision the effectiveness of interventions to promote mental wellbeing relative to other health related interventions.

It is essential that the preference-based measure adopted by an economic evaluation captures all

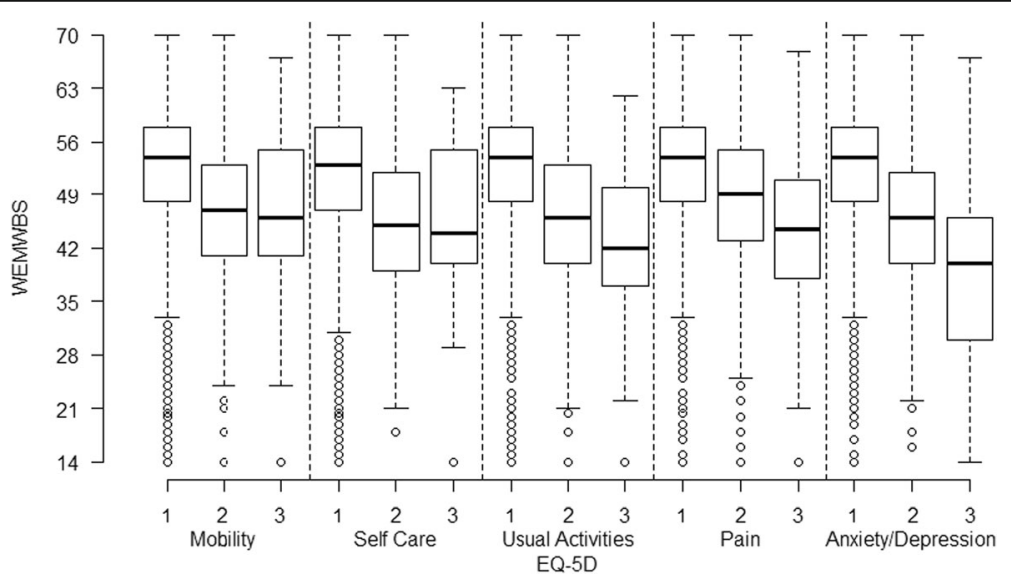

Fig. 2 Distribution of the WEMWBS scores for each level of each domain of EQ-5D-3L 


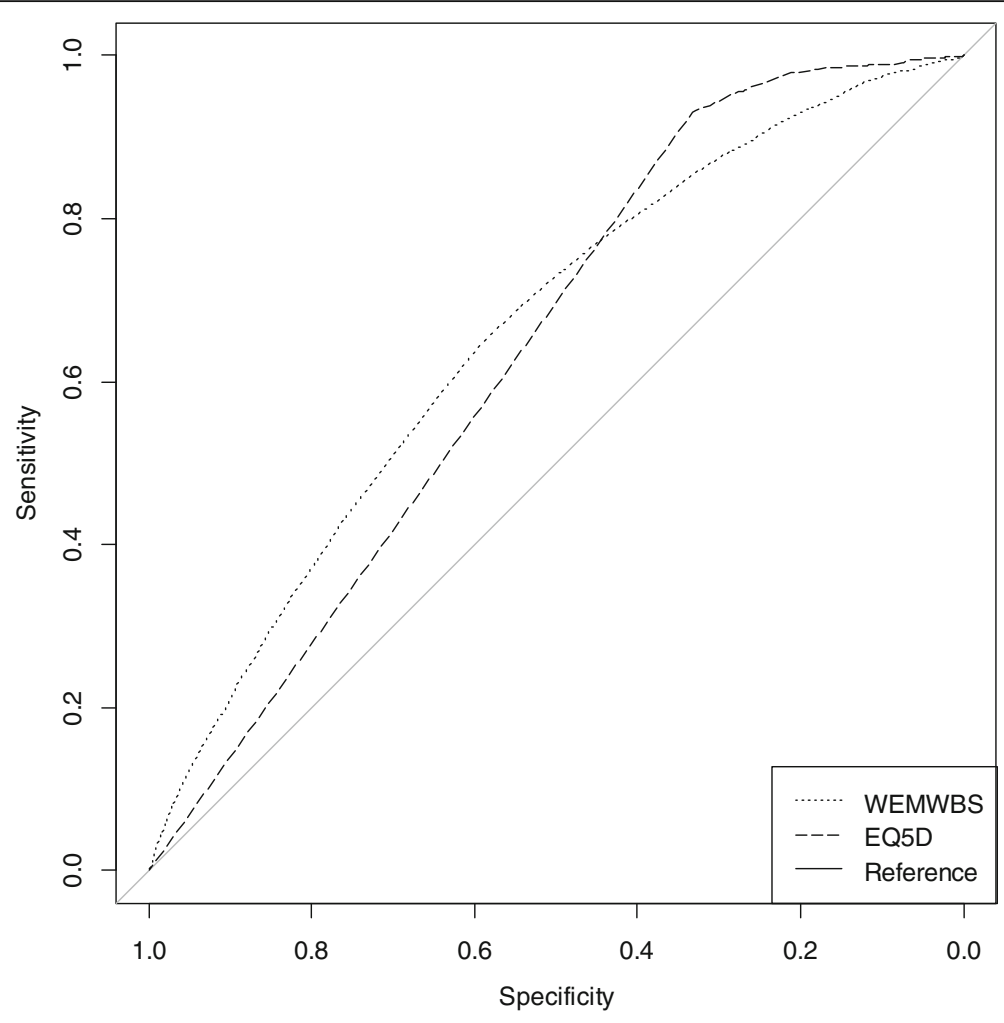

Fig. 3 Roc curves for WEMWBS and EQ-5D-3L predicting very good self-report health

consequences of the alternatives being evaluated that might materially affect the net benefit of each alternative to the decision-maker. It can be argued that wellbeing is a concept that extends existing concepts of health, prompting the need for a health-related measure of quality-of-life that will not underestimate the benefit of interventions that improve wellbeing. This remains an issue for interventions in sectors such as social care and education, and is increasingly relevant for public health and mental health interventions [27, 28]. The EQ-5D-3L has been shown to capture the impact of health care interventions for a broad range of conditions, but the fact that we found a ceiling effect in the EQ-5D-3L (as have others before us [51], with nearly three quarters of participants at the maximum score reinforces the likelihood that it does not capture relevant changes that matter to individuals or, therefore, to economic evaluations [51]. The EQ-5D-3L is preference-based, i.e. tariffs exist that

Table 4 Linear models of WEMWBS and EQ-5D-3L, and WEMWBS and EQ-5D-3L Visual Analogue Scale

\begin{tabular}{|c|c|c|c|c|c|c|}
\hline Model & & Estimate & $95 \% \mathrm{Cl}$ & & $p$-value & \\
\hline \multirow[t]{2}{*}{ EQ-5D-3L ${ }^{a}$} & Constant & 0.468 & 0.438 & 0.498 & $<0.001$ & \\
\hline & WEMWBS & 0.0082 & 0.0077 & 0.0088 & $<0.001$ & \\
\hline \multirow[t]{2}{*}{ EQ-5D-3L VAS ${ }^{b}$} & Constant & 36.4 & 33.9 & 38.8 & $<0.001$ & \\
\hline & WEMWBS & 0.786 & 0.740 & 0.832 & $<0.001$ & \\
\hline Model & ANOVA & Sum of Squares & Degrees of Freedom & Mean Square & F statistic & $p$-value \\
\hline \multirow[t]{3}{*}{$E Q-5 D-3 L^{a}$} & Regression & 38.86 & 1 & 38.86 & 846.84 & $<0.001$ \\
\hline & Residuals & 336.33 & 7329 & 0.046 & & \\
\hline & Total & 375.19 & 7330 & & $R^{2}$ & 0.104 \\
\hline \multirow[t]{3}{*}{ EQ-5D-3L VAS ${ }^{b}$} & Regression & 1 & 324278 & 324278 & 1110.8 & $<0.001$ \\
\hline & Residuals & 6768 & 1975775 & 292 & & \\
\hline & Total & 6769 & 2300053 & & $R^{2}$ & 0.141 \\
\hline
\end{tabular}


reflect societal preferences for different types of health gain, relative to life extension, to permit calculation of QALYs. If a similar tariff existed for WEMWBS that allowed estimation of Wellbeing Adjusted Life Year (WALYs) gained, this could be used to support prioritysetting within and across sectors in a way that reflects societal preferences more appropriately. Further research would be required to understand how the overlap between health and wellbeing varies in different populations, and to determine how the QALY and/or WALY can be used to value the benefits of interventions in these populations, while avoiding double-counting.

A limitation of our study is that we compared WEMWBS to the EQ-5D-3L. A new version (EQ-5D-5L) has recently been published [52] which has ameliorated some of the limitations of EQ-5D-3L discussed in this paper and has reduced ceiling effects with increased discriminatory power [53].

WEMWBS has the potential to be used as the basis of a preference-based measure to evaluate and prioritise public sector interventions between and within sectors, including traditional health related interventions. However, it has not yet been used to inform priority-setting and a preference-based tariff does not currently exist. The next step for our research is to develop and evaluate the utility of a well-being adjusted life year (WALY) based on WEMWBS. The stages in the development of the WALY will include a valuation exercise to generate a preference tariff for WEMWBS, the identification of an appropriate preference elicitation technique for wellbeing states, and exploration of the variation in valuations across samples.

\section{Conclusions}

There is wide variation in the levels of mental wellbeing amongst participants with very high levels of self-reported health who score at ceiling level on the EQ-5D-3L with evidence of limited mapping of WEMWBS onto EQ-5D-3L. These results suggest that the two measures examine related, but not identical, aspects of quality of life. We propose exploration of the feasibility, appropriateness, and practicality of a Wellbeing-Adjusted Life Year.

\begin{abstract}
Acknowledgements
Rebecca Johnson, David Jenkinson, Chris Stinton and Aileen Clarke are supported by the National Institute for Health Research Collaboration for Leadership in Applied Health Research and Care West Midlands at University Hospitals Birmingham NHS Foundation Trust. Sian Taylor-Phillips is supported by a clinical trials fellowship from the National Institute for Health Research (NIHR). The views expressed in this publication are those of the authors and not necessarily those of the NHS, the National Institute for Health Research or the Department of Health. Any errors are the responsibility of the authors.
\end{abstract}

\section{Authors' contributions}

RJ jointly developed the idea for the work and conducted the original data collection and analysis on which the work is based. She advised on design and analysis and commented on initial and final drafts of the paper. DJ cleaned and analysed the data. He commented on initial and final drafts of the paper. CS analysed the data and wrote the paper. STP cleaned and analysed the data. She commented on initial and final drafts of the paper. JM commented on initial and final drafts of the paper. SSB developed the WEMWBS measure and the body of research now underpinning it. She advised on design and analysis and read final drafts of the paper. AC jointly developed the idea for the work and provided the funding. She advised on design and analysis and commented on initial and final drafts of the paper. All authors read and approved the final manuscript.

\section{Competing interests}

RJ, DJ, CS, STP, JM, and AC reports no competing interests.

SSB led the development and validation of WEMWBS.

\section{Ethics approval and consent to participate}

Ethical approval for secondary analysis with an existing database was obtained from the University of Warwick Biomedical Sciences and Research Ethics Committee, REGO-2015-1500.

Received: 29 August 2015 Accepted: 6 September 2016

Published online: 08 September 2016

\section{References}

1. EuroQoL Group. EuroQoL: a new facility for the measurement of health-related quality of life. Health Policy. 1990;16:199-208.

2. Brooks R. EuroQol: the current state of play. Health policy. 1996;37:53-72.

3. Bharmal M, Thomas J. Comparing the EQ-5D and the SF-6D descriptive systems to assess their ceiling effects in the US general population. Value Health. 2006;9(4):262-71.

4. Johnson JA, Coons SJ. Comparison of the EQ-5D and SF-12 in an adult US sample. Qual Life Res. 1998;7(2):155-66.

5. Barton GR, Bankart J, Davis AC, Summerfield QA. Comparing utility scores before and after hearing-aid provision. Appl Health Econ Health Policy. 2004;3(2):103-5.

6. Papaioannou D, Brazier J, Parry G. How valid and responsive are generic health status measures, such as EQ-5D and SF-36, in schizophrenia? A systematic review. Value Health. 2011;14(6):907-20.

7. Saarni SI, Viertio S, la Pera J, Koskinen S, Lonnqvist J, Suvisaari J. Quality of life of people with schizophrenia, bipolar disorder and other psychotic disorders. Br J Psychiatry. 2010;197:386-94.

8. Tosh J, Brazier J, Evans P, Longworth L. A review of generic preferencebased measures of health-related quality of life in visual disorders. Value Health. 2011;15(1):118-27.

9. Brazier J. Is the EQ-5D fit for purpose in mental health? Br J Psychiatry. 2010;197:348-9.

10. Chida Y, Steptoe A. Positive psychological well-being and mortality: a quantitative review of prospective observational studies. Psychosom Med. 2008;70(7):741-56

11. Haro JM, Ayuso-Mateos JL, Bitter I, Demotes-Mainard J, Leboyer M, Lewis SW, et al. ROAMER: a European roadmap for mental health research. Int J Methods Psychiatr Res. 2014;23:1-13.

12. Williams $A$. The measurement and valuation of health: a chronicle. York: University of York; 1995. http://www.york.ac.uk/che/pdf/DP136.pdf. Accessed 20 March 2015.

13. Dolan P. Modeling valuations for EuroQol health states. Med Care. 1997;35:1095-108.

14. Brazier J, Roberts J, Tsuchiya A, Busschbach J. A comparison of the EQ-5D and SF-6D across seven patient groups. Health Econ. 2004;13:873-84.

15. Tennant R, Hiller L, Fishwick R, Platt $S$, Joseph $S$, Weich $S$, et al. The Warwick-Edinburgh mental well-being scale (WEMWBS): development and UK validation. Health Qual Life Outcomes. 2007;5:63.

16. Clarke A, Friede T, Putz R, Ashdown J, Martin S, Blake A, et al. Warwick-Edinburgh Mental Well-being Scale (WEMWBS): validated for teenage school students in England and Scotland. A mixed methods assessment. BMC Public Health. 2011;11:487.

17. Maheswaran H, Weich S, Powell J, Stewart-Brown S. Evaluating the responsiveness of the Warwick Edinburgh Mental Well-Being Scale (WEMWBS): Group and individual level analysis. Health Qual Life Outcomes. 2012;10:156

18. Taggart F, Friede T, Weich S, Clarke A, Johnson M, Stewart-Brown S. Cross cultural evaluation of the Warwick-Edinburgh Mental Well-being Scale 
(WEMWBS) - a mixed methods study. Health Qual Life Outcomes. 2013;11:27. doi:10.1186/1477-7525-11-27.

19. Braiden HJ, McDaniel B, Duffy J, McCann M. A pilot study of the Incredible Years BASIC parenting programme with bereaved families. J Child Serv. 2011;6:141-55

20. Collins J, Gibson A, Parkin S, Parkinson R, Shave D, Dyer C. Counselling in the workplace: How time-limited counselling can effect change in wellbeing. Couns Psychother Res. 2012;12:84-92.

21. Crone DM, O'Connell EE, Tyson PJ, Clark-Stone F, Opher S, James DV. 'Art Lift' intervention to improve mental well-being: An observational study from UK general practice. Int J Ment Health Nurs. 2013;22:279-86.

22. Huppert FA, Johnson DM. A controlled trial of mindfulness training in schools: The importance of practice for an impact on well-being. J Posit Psychol. 2010;5:264-74.

23. Lindsay $G$, Strand $S$, Davis H. A comparison of the effectiveness of three parenting programmes in improving parenting skills, parent mental-well being and children's behaviour when implemented on a large scale in community settings in 18 English local authorities: the parenting early intervention pathfinder (PEIP). BMC Public Health. 2011:11:962.

24. Powell J, Hamborg T, Stallard N, Burls A, McSorley J, Bennett K, et al. Effectiveness of a web-based cognitive-behavioral tool to improve mental well-being in the general population: randomized controlled trial. J Med Internet Res. 2013;15:e2.

25. Odou N, Vella-Brodrick DA. The efficacy of positive psychology interventions to increase well-being and the role of mental imagery ability. Soc Indic Res. 2013;110:111-29.

26. Crawford MJ, Robotham D, Thana L, Patterson S, Weaver T, Barber R, Wykes T, Rose D. Selecting outcome measures in mental health: the views of service users. J Ment Health. 2011;20(4):336-46.

27. Scottish health outcomes framework accessible at: http://www.healthscotland. com/scotlands-health/population/mental-health-indicators.aspx. Accessed 20 July 2015.

28. Public Health England. $(2011,2013)$. Improving outcomes and supporting transparency. https://www.gov.uk/government/publications/healthy-liveshealthy-people-improving-outcomes-and-supporting-transparency. Accessed 02 June 2015

29. Longworth L, Rowen D. Mapping to obtain EQ-5D utility values for use in NICE health technology assessments. Value Health. 2013;16:202-10.

30. Public Health England. Coventry Health Profile. West Midlands Public Health Observatory. 2014

31. Putz R, Clarke A, Stewart-Brown S. The Coventry Wellbeing Report. Internal Report for Coventry Partnership. 2011. at: http://www2.warwick.ac.uk/fac/ med/research/platform/wemwbs/. Accessed 20 March 2015.

32. Putz R, Clarke A, Stewart-Brown S. The Coventry Wellbeing Report. Internal Report for Coventry Partnership. 2012. at: http://www2.warwick.ac.uk/fac/ med/research/platform/wemwbs/. Accessed 20 March 2015.

33. Johnson R, Clarke A, Stewart-Brown S. The Coventry Wellbeing Report Internal Report for Coventry City Council Department of Public Health; 2013. at: http://www2.warwick.ac.uk/fac/med/research/platform/wemwbs/. Accessed 20 March 2015.

34. Hoffmeyer-Zlotnik JHP. New sampling designs and the quality of data. Developments in applied statistics. In: Ferligoj A, Mrvar A, editors. Developments in Applied Statistics. Ljubljana: FDV Methodoloski zvezki; 2003. p. 205-17.

35. van Agt HM, Essink-Bot ML, Krabbe PF, Bonsel GJ. Test-retest reliability of health state valuations collected with the EuroQol questionnaire. Soc Sci Med. 1994:39(11):1537-44.

36. Kind P, Dolan P, Gudex C, Williams A. Variations in population health status: results from a United Kingdom national questionnaire survey. BMJ. 1998;316(7133):736-41.

37. Kind P, Hardman G, Macran S. UK population norms for EQ-5D. York: Centre for Health Economics, University of York; 1999.

38. Hurst NP, Jobanputra P, Hunter M, Lambert M, Lochhead A, Brown $\mathrm{H}$. Validity of Euroqol-a generic health status instrument-in patients with rheumatoid arthritis economic and health outcomes research group. Rheumatology. 1994;33(7):655-62.

39. Rabin R, Charro FD. EQ-SD: a measure of health status from the EuroQol Group. Ann Med. 2001:33(5):337-43.

40. Paulhus DL. Balanced inventory of desirable responding (BIDR). Acceptance and Commitment Therapy. Measures Package. 1988:41
41. Watson D, Clark LA, Tellegen A. Development and validation of brief measures of positive and negative affect: the PANAS scales. J Pers Soc Psychol. 1988;54(6):1063.

42. Eriksson I, Undén AL, Elofsson S. Self-rated health. Comparisons between three different measures. Results from a population study. International Journal of Epidemiology. 2001;30(2):326-33.

43. Zajacova A, Dowd JB. Reliability of self-rated health in US adults. Am J Epidemiol. 2011;174(8):977-83.

44. Idler EL, Benyamini Y. Self-rated health and mortality: a review of twenty-seven community studies. J Health Soc Behav. 1997;38:21-37.

45. Appels A, Bosma H, Grabauskas V, Gostautas A, Sturmans F. Self-rated health and mortality in a Lithunian and a Dutch population. Soc Sci Med. 1996;28:681-90.

46. DeSalvo KB, Bloser N, Reynolds K, He J, Muntner P. Mortality prediction with a single general self-rated health question. J Gen Intern Med. 2006;21(3):267-75.

47. Kaplan MS, Berthelot JM, Feeny D, McFarland BH, Khan S, Orpana H. The predictive validity of health-related quality of life measures: mortality in a longitudinal population-based study. Qual Life Res. 2007;16(9):1539-46.

48. Schnittker J, Bacak V. The increasing predictive validity of self-rated health. PLoS One. 2014;9(1):e84933.

49. R Core Team. R: A language and environment for statistical computing. Vienna: R Foundation for Statistical Computing; 2013. http://www.R-project.org/.

50. Robin X, Turck N, Hainard A, Tiberti N, Lisacek F, Sanchez JC, et al. pROC: an open-source package for R and $\mathrm{S}+$ to analyze and compare ROC curves. BMC Bioinformatics. 2011;12:77

51. Longworth L, Yang Y, Young T, Hernandez Alva M, Mukuria C, Rowen D, et al. Use of generic and condition-specific measures of health related quality of life in NICE decision-making: systematic review, statistical modelling and survey. Health Technol Assess. 2014;18(9):1-224.

52. Herdman M, Gudex C, Lloyd A, Janssen M, Kind P, Parkin D, et al. Development and preliminary testing of the new five-level version of EQ-5D (EQ-5D-5L). Qual Life Res. 2011;20:1727-36.

53. Janseen MF, Pickard AS, Golicki D, Gudex C, Niewada M, Scalone L, et al. Measurement properties of the EQ-5D-5L compared to the EQ-5D-3L across eight patient group: a multi-country study. Qual Life Res. 2013;22:1717-27.

\section{Submit your next manuscript to BioMed Central and we will help you at every step:}

- We accept pre-submission inquiries

- Our selector tool helps you to find the most relevant journal

- We provide round the clock customer support

- Convenient online submission

- Thorough peer review

- Inclusion in PubMed and all major indexing services

- Maximum visibility for your research

Submit your manuscript at www.biomedcentral.com/submit 